\title{
NUMERICAL INTEGRATION ON THE SPHERE
}

\author{
KENDALL ATKINSON
}

(Received 28 October 1980)

(Revised 1 February 1981)

\begin{abstract}
This is a discussion of some numerical integration methods for surface integrals over the unit sphere in $\mathbf{R}^{3}$. Product Gaussian quadrature and finite-element type methods are considered. The paper concludes with a discussion of the evaluation of singular double layer integrals arising in potential theory.
\end{abstract}

\section{Introduction}

This is a discussion of some numerical integration methods for the surface integral

$$
I(f)=\int_{U} f(Q) d \sigma
$$

with $U$ the unit sphere in $\mathbf{R}^{3}$. The integration formulae will include product Gaussian quadrature and some methods based on breaking $U$ into smaller triangular elements with various associated low order integration schemes.

The motivation for discussing such methods arises from the desire to solve integral equations defined over simple smooth surfaces $S$ in $\mathbf{R}^{3}$,

$$
\lambda \rho(P)-\int_{S} K(P, Q) \rho(Q) d \sigma(Q)=\psi(P), \quad P \in S .
$$

Such equations can arise in a variety of applications, although we are particularly interested in those equations arising from solving potential theory problems in $\mathbf{R}^{3}$. To deal with integrals over a general surface $S$, we assume there is a smooth 1-1 mapping of $U$ onto $S$; then an integral over $S$ can be transformed into one of the form (1.1).

(c) Copyright Australian Mathematical Society 1982. 
The product Gaussian quadrature formula is discussed and illustrated in Section 2. Methods for triangulating the sphere and some associated integration formulas are given in Section 3. Since most kernel functions $K(P, Q)$, in (1.2), are singular in potential theory applications, we discuss the evaluation of one such integral in Section 4.

For a review of integration methods on the sphere, see Keast and Diaz [6], Lebedev [7], and Stroud [13, Sections 2.6 and 8.4]. The methods discussed in the present paper are not optimal, but they are well-suited to the solution of integral equations. Moreover, the theory of optimal methods is far from complete, as has been noted in [7]; consequently it would not be possible to carry out a complete error analysis of the resulting numerical methods for solving (1.2).

\section{Product Gaussian quadrature}

Let $I(f)$ be written using spherical coordinates

$$
I(f)=\int_{0}^{2 \pi} \int_{0}^{\pi} F(\theta, \phi) \sin \theta d \theta d \phi
$$

with $f(\theta, \phi) \equiv f(x, y, z)$. The integral is approximated by

$$
I_{m}(f)=\frac{\pi}{m} \sum_{j=1}^{2 m} \sum_{i=1}^{m} w_{i} f\left(\theta_{i}, \phi_{j}\right)
$$

The $\left\{\theta_{i}\right\}$ are chosen so that $\left\{\cos \left(\theta_{i}\right)\right\}$ and $\left\{w_{i}\right\}$ are the Gauss-Legendre nodes and weights on $[-1,1]$. The points $\phi_{j}$ are evenly spaced on $[0,2 \pi]$ with spacing $\pi / m$; usually

$$
\phi_{j}=j \pi / m \quad \text { or } \quad\left(j-\frac{1}{2}\right) \pi / m \text {. }
$$

With this choice of node points and weights, $I_{m}(f)$ integrates exactly any polynomial $f(x, y, z)$ of degree less than $2 m$; see [13, page 40] for a proof. For an integration formula, the degree of precision is $n$ if the formula is exact for some polynomial of degree $n+1$. Hence $I_{m}(f)$ has degree of precision $2 m-1$.

The formula $I_{m}(f)$ is less efficient than the optimal formulae of [7], but not badly so. For methods of an increasing degree of precision, Lebedev introduces the efficiency index

$$
\eta(n)=(n+1)^{2} /(3 N(n))
$$

where $n$ is the degree of precision and $N(n)$ the number of associated node points on $U$. The larger the index for a given $n$, the more efficient is the method. The formulae developed by Lebedev satisfy $\eta(n) \rightarrow 1$ as $n \rightarrow \infty$. The above Gaussian formula has index $\eta(2 m-1)=2 / 3$ for $m>1$. Lebedev's formulae use only $2 / 3$ the number of node points used by $I_{m}(f)$. This is not a large difference and it is offset somewhat by the ease with which $I_{m}(f)$ is constructed. 
Convergence results for $I_{m}(f)$ can be obtained from an approximation theorem of Ragozin [11]. Using this, it is straightforward to show that, if $f(x, y, z)$ is $k$ times continuously differentiable on $U$, then

$$
\left|I(f)-I_{m}(f)\right|<C /\left((2 m-1)^{k}\right) \quad \text { for } m>1 .
$$

This bound shows the same rapid convergence associated with Gaussian quadrature for one variable integration. A short proof of (2.5) is given in [1].

EXAMPLES. Four numerical examples will be given, and they will be referenced by the four different surfaces $S_{i}$ that are used. The first two examples are

$$
\int_{S_{i}} e^{x} d \sigma, \quad i=1,2
$$

with $S_{i}$ the ellipsoidal surface $(x / a)^{2}+(y / b)^{2}+(z / c)^{2}=1$, where $S_{1}$ uses $(a, b, c)=(1,1,2)$ and $S_{2}$ uses $(1,2,5)$. The last two examples are to calculate the surface area of $S_{i}$,

$$
\int_{S_{i}} d \sigma, \quad i=3,4
$$

with $S_{i}$ a 'peanut-shaped' region given by

$$
(x, y, z)=R(\theta)(a \cos \phi \sin \theta, b \sin \phi \sin \theta, \cos \theta)
$$

where

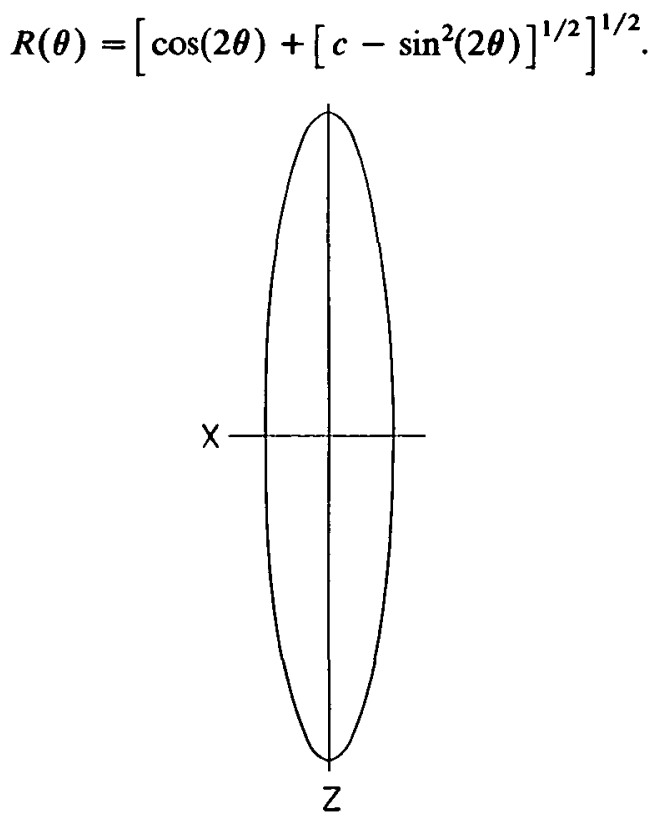

Figure 1. Crass-section of the ellipsoid $S_{2}$. 


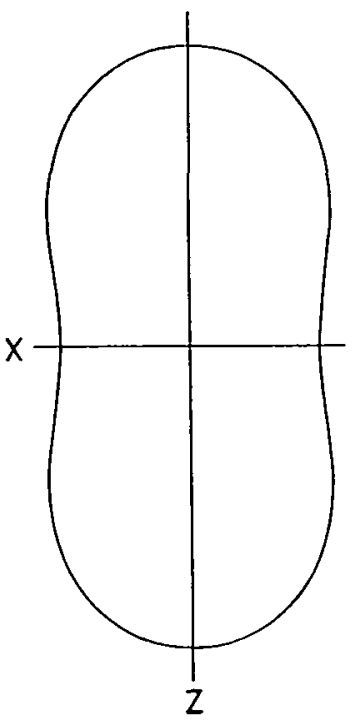

Figure 2. Cross-section of the surface $S_{3}$.

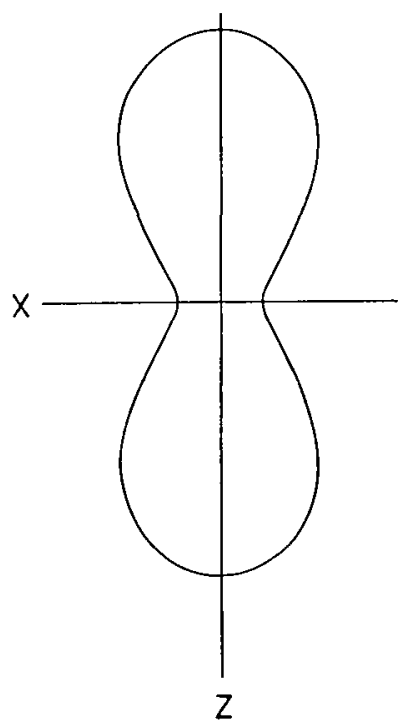

Figure 3. Cross-section of the surface $S_{4}$.

Surface $S_{3}$ uses $(a, b, c)=(1,2,2)$ and $S_{4}$ uses $(1,2,1.1)$. The cross-sections in the $x, z$-plane of the surfaces $S_{2}, S_{3}$, and $S_{4}$ are shown in Figures 1 to 3. Surfaces $S_{2}$ and $S_{4}$ are somewhat more ill-behaved than $S_{1}$ and $S_{3}$.

Table 1 contains the numerical results for the four integrals. The column $n$ gives the number of integration nodes. As expected, the convergence is rapid. 
TABLE 1

Errors for product Gaussian quadrature

\begin{tabular}{|rr|c|c|c|c|}
\hline & & \multicolumn{4}{|c|}{ Relative error for the integration over $S_{i}$} \\
\hline$m$ & $n$ & $i=1$ & $i=2$ & $i=3$ & $i=4$ \\
\hline 4 & 32 & $-3.0 \mathrm{E}-4$ & $-1.5 \mathrm{E}-3$ & $7.8 \mathrm{E}-3$ & $-3.4 \mathrm{E}-2$ \\
8 & 128 & $-1.3 \mathrm{E}-6$ & $-8.6 \mathrm{~F}-5$ & $1.4 \mathrm{E}-4$ & $-2.2 \mathrm{E}-2$ \\
12 & 288 & $-9.0 \mathrm{E}-9$ & $-7.3 \mathrm{E}-6$ & $-3.3 \mathrm{E}-6$ & $-5.9 \mathrm{E}-3$ \\
16 & 512 & $<5.0 \mathrm{E}-12$ & $-7.9 \mathrm{E}-7$ & $-3.7 \mathrm{E}-7$ & $-1.4 \mathrm{E}-3$ \\
20 & 800 & $<5.0 \mathrm{E}-12$ & $-9.9 \mathrm{E}-8$ & $-1.2 \mathrm{E}-8$ & $-3.7 \mathrm{E}-4$ \\
\hline
\end{tabular}

\section{Finite element integration}

Letting $\left\{\Delta_{1}, \ldots, \Delta_{n}\right\}$ be a triangulation of $U$, we can write

$$
I(f)=\sum_{j=1}^{n} \int_{\Delta,} f(Q) d \sigma
$$

We will consider numerical approximations to $I(f)$ based on approximating each integral over $\Delta_{j}$ using a fixed low order integration rule. First, we discuss how to triangulate $U$.

The usual manner of subdividing $U$ is based on using a rectangular or triangular grid on the rectangle $\{(\theta, \phi) \mid 0 \leqslant \theta \leqslant \pi, 0 \leqslant \phi<2 \pi\}$, and this is mapped onto a triangulation of $U$ using the standard spherical coordinates formula. The advantage of this method is its simplicity, and usually it is rapid to implement. The main disadvantage is that it results in a very nonuniform distribution of nodes and elements on $U$; usually there are relatively more nodes near the poles $z= \pm 1$. The elements are also quite varied in shape and size, in general. For these reasons, we consider another method of subdivision.

Create an initial triangulation of $U$ by inscribing a tetrahedron, octahedron, or icosahedron inside $U$, and then project it outward onto the surface $U$. This gives a uniform subdivision of equilateral spherical triangles, with 4,8 , or 20 faces, respectively. To subdivide an existing triangulation $\left\{\Delta_{i}\right\}$, we divide each face $\Delta_{i}$ into four smaller triangles: find the midpoints of the sides of $\Delta_{i}$, and then connect them by great circle paths. If $\Delta_{i}$ is not too large, then the four new triangles created from $\Delta_{i}$ will be almost congruent, and will be nearly similar to $\Delta_{i}$. This triangulation method leads to a fairly uniform subdivision of $U$, particularly when the initial subdivision is an icosahedron. Denote the three triangulation schemes by $T_{t, n}, T_{o, n}$, and $T_{i, n}$, depending on whether the initial 
triangulation uses a tetrahedron, octahedron, or icosahedron, The subscript $n$ indicates the number of faces.

THE CENTROID RULE. The simplest method for estimating the integral over $\Delta_{i}$ is

$$
\int_{\Delta_{i}} f(Q) d \sigma \approx f\left(Q_{i}\right) \cdot \operatorname{Area}\left(\Delta_{i}\right)
$$

where $Q_{i}$ is the centroid of $\Delta_{i}$. If $v_{1}, v_{2}$ and $v_{3}$ denote the vertices of $\Delta_{i}$, define

$$
Q_{i}=\left(v_{1}+v_{2}+v_{3}\right) /\left|v_{1}+v_{2}+v_{3}\right| \text {. }
$$

Using this in (3.1), we obtain

$$
I(f) \approx \sum_{i=1}^{n} f\left(Q_{i}\right) \operatorname{Area}\left(\Delta_{i}\right) \equiv C_{n}(f)
$$

and we shall call this the 'centroid rule'. This simple rule is surprisingly accurate, especially when certain triangulations of $U$ are being used.

If $f(Q)$ is twice continuously differentiable on $U$, then a bound on the rate of convergence is given by

$$
\left|I(f)-C_{n}(f)\right| \leqslant k_{1} / n \text {. }
$$

A proof is sketched later in this section. The numerical results given below also seem to confirm the correctness of the order.

Nonetheless, the method has another interesting aspect. Table 2 gives the degree of precision $d$ of $C_{n}(f)$ for the various polyhedral triangulation schemes. These are somewhat surprising results for such a simple method. Clearly, this triangulation method is important, as other nonuniform triangulations generally have only degree of precision 0 or 1 . The results of Table 2 can be proved in a straightforward way, based on the results of [12].

\section{TABLE 2}

Degree of precision of the centroid rule

\begin{tabular}{|l|ccc|}
\hline Triangulation & $T_{t, n}$ & $T_{o, n}$ & $T_{i, n}$ \\
\hline Degree of Precision & 2 & 3 & 5 \\
\hline
\end{tabular}

EXAMPLES. We use the integrals (2.6) and (2.7) that were used for the product Gaussian quadrature. The triangulation method is $T_{i, n}$, and the results are given in Tables 3 and 4. 
TABLE 3

Numerical examples 1 and 2 for the centroid rule

\begin{tabular}{|r|r|r|r|r|}
\hline & \multicolumn{2}{|c|}{ Surface $S_{1}$} & \multicolumn{2}{c|}{ Surface $S_{2}$} \\
\hline 20 & $\begin{array}{l}\text { Relative } \\
\text { error }\end{array}$ & Ratio & $\begin{array}{l}\text { Relative } \\
\text { error }\end{array}$ & Ratio \\
\hline 80 & $-1.2 \mathrm{E}-3$ & -10 & $-4.7 \mathrm{E}-3$ & 66 \\
320 & $1.1 \mathrm{E}-5$ & 5.9 & $-7.0 \mathrm{E}-5$ & -4.10 \\
1280 & $3.6 \mathrm{E}-7$ & 5.1 & $1.8 \mathrm{E}-5$ & 4.6 \\
\hline
\end{tabular}

TABLE 4

Numerical examples 3 and 4 for the centroid rule

\begin{tabular}{|r|r|r|r|r|}
\hline & \multicolumn{2}{|c|}{ Surface $S_{3}$} & \multicolumn{2}{c|}{ Surface $S_{4}$} \\
\hline$n$ & $\begin{array}{l}\text { Relative } \\
\text { error }\end{array}$ & Ratio & $\begin{array}{l}\text { Relative } \\
\text { error }\end{array}$ & Ratio \\
\hline 20 & $-1.1 \mathrm{E}-2$ & 6.4 & $-2.8 \mathrm{E}-1$ & 75 \\
80 & $-1.7 \mathrm{E}-3$ & 118 & $-3.8 \mathrm{E}-3$ & -2.2 \\
320 & $-1.5 \mathrm{E}-5$ & 2.2 & $1.7 \mathrm{E}-3$ & -16 \\
1280 & $-6.8 \mathrm{E}-6$ & & $-1.0 \mathrm{E}-4$ & \\
\hline
\end{tabular}

With most examples, including the ones shown here, the error first decreases very rapidly and then settles down to a slower rate of decrease, usually one more consistent with the theoretical bound of $O(1 / n)$. When the triangulations $T_{t, n}$ and $T_{o, n}$ are used, similar behaviour holds, but the errors are not as small as with $T_{i, n}$.

AN ISOPARAMETRIC METHOD. We use the isoparametric mapping approach of the finite element method. Let $\sigma_{0}$ denote the unit simplex in $\mathbf{R}^{2}, \sigma_{0}=\{(s, t) \mid 0 \leqslant$ $s, t, s+t \leqslant 1\}$. Let $\Delta_{i}$ be a spherical triangle with vertices $v_{1}, v_{2}$ and $v_{3}$. Define

$$
p(s, t)=v_{1}+t\left(v_{2}-v_{1}\right)+s\left(v_{3}-v_{1}\right)
$$


and

$$
q(s, t)=p(s, t) /|p(s, t)|, \quad \text { for }(s, t) \in \sigma_{0} .
$$

It is straightforward to show that $p$ is a 1-1 mapping of $\sigma_{0}$ onto the planar triangle determined by $v_{1}, v_{2}$ and $v_{3}$ and that $q$ is a 1-1 mapping of $\sigma_{0}$ onto $\Delta_{i}$.

Using (3.6), we can write

$$
\int_{\Delta_{\imath}} f(Q) d \sigma=\int_{\sigma_{0}} f(q(s, t))\left|q_{s} \times q_{\imath}\right| d s d t
$$

with $q_{s}$ and $q_{t}$ denoting the partial derivatives of $q(s, t)$. We now integrate this numerically with an integration method of degree of precision 3 :

$$
\begin{aligned}
\int_{\sigma_{0}} g(s, t) d s d t \approx & \frac{1}{40}[g(0,0)+g(1,0)+g(0,1)] \\
& +\frac{1}{15}\left[g\left(0, \frac{1}{2}\right)+g\left(\frac{1}{2}, 0\right)+g\left(\frac{1}{2}, \frac{1}{2}\right)\right]+\frac{9}{40} g\left(\frac{1}{3}, \frac{1}{3}\right) .
\end{aligned}
$$

Under the mapping $q$, the points $\{(0,0),(0,1),(1,0)\}$ map into $v_{1}, v_{2}$ and $v_{3}$, the points $\left\{\left(\frac{1}{2}, 0\right),\left(0, \frac{1}{2}\right),\left(\frac{1}{2}, \frac{1}{2}\right)\right\}$ map into the centroid $Q_{i}$ of $\Delta_{i}$. We apply (3.8) to the right side of (3.7) for each $\Delta_{i}$ and call the sum of the resulting numerical integrals $J_{n}(f)$.

For the convergence of $J_{n}(f)$, we show later that, if $f(x, y, z)$ is four times continuously differentiable on $U$, then

$$
\left|I(f)-J_{n}(f)\right|<k_{2} / n^{2}
$$

Thus this method should be an improvement on the centroid method. In actual examples, however, the centroid method has almost always been much superior, provided $T_{i, n}$ was used with $n<1280$, which was the bound on $n$ in most computations.

EXAmples. As before, we evaluate the integrals (2.6) and (2.7), and use $T_{i, n}$. The results are given in Tables 5 and 6, where 'Nodes' gives the number of integration node points on $U$. The work with $J_{n}(f)$ is about the same as for $C_{4 n}(f)$, since the triangulation $T_{i, 4 n}$ is needed to obtain the nodes used in $J_{n}(f)$. 
TABLE 5

Numerical examples 1 and 2 for the isoparametric method

\begin{tabular}{|rl|l|l|l|r|}
\hline & & \multicolumn{2}{|c|}{ Surface $S_{1}$} & \multicolumn{2}{c|}{ Surface $S_{2}$} \\
\hline$n$ & Nodes & $\begin{array}{l}\text { Relative } \\
\text { error }\end{array}$ & Ratio & $\begin{array}{l}\text { Relative } \\
\text { error }\end{array}$ & Ratio \\
\hline 20 & 62 & $-2.8 \mathrm{E}-2$ & 13.7 & $-3.0 \mathrm{E}-2$ & 14.3 \\
80 & 242 & $-2.0 \mathrm{E}-3$ & 15.3 & $-2.1 \mathrm{E}-3$ & 16.0 \\
320 & 962 & $-1.3 \mathrm{E}-4$ & & $-1.3 \mathrm{E}-4$ & \\
\hline
\end{tabular}

TABLE 6

Numerical examples 3 and 4 for the isoparametric method

\begin{tabular}{|rl|l|r|r|r|}
\hline & \multicolumn{2}{|c|}{ Surface $S_{3}$} & \multicolumn{2}{c|}{ Surface $S_{4}$} \\
\hline$n$ & Nodes & $\begin{array}{l}\text { Relative } \\
\text { error }\end{array}$ & Ratio & $\begin{array}{l}\text { Relative } \\
\text { error }\end{array}$ & Ratio \\
\hline 20 & 62 & $-3.2 \mathrm{E}-2$ & 11 & $-1.8 \mathrm{E}-1$ & 31 \\
80 & 242 & $-2.9 \mathrm{E}-3$ & 12 & $-5.8 \mathrm{E}-3$ & -6.9 \\
320 & 962 & $-1.3 \mathrm{E}-4$ & & $8.4 \mathrm{E}-4$ & \\
\hline
\end{tabular}

Based on our examples, the centroid rule should be used in preference to the isoparametric method $J_{n}(f)$, provided $n$ is not too large and $T_{i, n}$ is used. With other values of $n$ or other triangulations, $J_{n}(f)$ is much more competitive, and possibly superior. Comparing with the other examples for the product Gaussian quadrature, the latter is generally superior in accuracy to $C_{n}(f)$, especially at moderate to high error tolerances.

ERror BoUnd DERIVATIONS. We will give only a sketch of the proof of (3.9); the details are straightforward, but algebraically complicated. First, note that the integration rule (3.8) is exact for all polynomials $g(s, t)$ of degree $<3$. For a general differentiable function $g(s, t)$, an error formula can be found in the standard way: expand $g(s, t)$ in a Taylor series through the third degree plus a remainder term, and apply the error functional for (3.8) to this equation. The error will be proportional to the fourth derivatives of $g$. 
To apply this to deriving (3.9), first assume that $f(x, y, z)$ is defined in a neighborhood of $U$, with continuous and bounded fourth order derivatives. Since (3.8) is applied to (3.7), consider the fourth derivative of the integrand in (3.7). It can be shown that

$$
\left|\frac{\partial^{4}}{\partial s^{k} \partial t^{4-k}}\left[f(q(s, t))\left|q_{s} \times q_{t}\right|\right]\right| \leqslant C \max \left\{\left|v_{1}-v_{2}\right|^{6},\left|v_{1}-v_{3}\right|^{6}\right\} .
$$

In addition, the following results can be shown for our triangulations $T_{i, n}, T_{o, n}$, and $T_{t, n}$ :

$$
c_{1} / n \leqslant \operatorname{Area}\left(\Delta_{i}\right) \leqslant c_{2} / 2, \quad \text { for } i=1, \ldots, n,
$$

where $c_{1}$ and $c_{2}>0$ and independent of $n$; furthermore,

$$
\begin{aligned}
c_{3} \max \left\{\left|v_{1}-v_{2}\right|^{2},\right. & \left.\left|v_{2}-v_{3}\right|^{2},\left|v_{3}-v_{1}\right|^{2}\right\} \leqslant \operatorname{Area}\left(\Delta_{i}\right) \\
& \leqslant c_{4} \min \left\{\left|v_{1}-v_{2}\right|^{2},\left|v_{2}-v_{3}\right|^{2},\left|v_{3}-v_{1}\right|^{2}\right\},
\end{aligned}
$$

with $i=1, \ldots, n, v_{1}, v_{2}$ and $v_{3}$ the vertices of $\Delta_{i}$, and $c_{3}$ and $c_{4}>0$ independent of $n$. Combining all of these results with the error formula for (3.8), derived using a Taylor series, we obtain (3.9).

For functions $f(Q)$ defined only on $U$, if they are four times differentiable using a local parametrization on $U$, then they can be extended to a new function on a neighborhood of $U$; and the extensions can be chosen to have the same degree of differentiability. For a discussion of this, see $[4$, pages 13,100$]$.

The proof of (3.5) is quite similar. We write

$$
\begin{aligned}
e_{i}= & \int_{\Delta_{i}} f(Q) d \sigma-f\left(Q_{i}\right) \operatorname{Area}\left(\Delta_{i}\right) \\
= & \int_{\sigma_{0}} f(q(s, t))\left|q_{s} \times q_{t}\right| d s d t-\frac{1}{2} f\left(q\left(\frac{1}{3}, \frac{1}{3}\right)\right)\left|q_{s}\left(\frac{1}{3}, \frac{1}{3}\right) \times q_{t}\left(\frac{1}{3}, \frac{1}{3}\right)\right| \\
& +f\left(Q_{i}\right)\left[\frac{1}{2}\left|q_{s}\left(\frac{1}{3}, \frac{1}{3}\right) \times q_{t}\left(\frac{1}{3}, \frac{1}{3}\right)\right|-\int_{\sigma_{0}}\left|q_{s} \times q_{t}\right| d s d t\right] \\
= & E_{i}^{(1)}+E_{i}^{(2)} .
\end{aligned}
$$

The point $\left(\frac{1}{3}, \frac{1}{3}\right)$ is the centroid of $\sigma_{0}$, and $Q_{i}=q\left(\frac{1}{3}, \frac{1}{3}\right)$. The integration rule

$$
\int_{\sigma_{0}} g(s, t) d s d t \approx \frac{1}{2} g\left(\frac{1}{3}, \frac{1}{3}\right)
$$

has degree of precision 1. Using the same kind of proof as that given for (3.9), it follows that

$$
E_{i}^{(1)}=O\left(1 / n^{2}\right)
$$


For $E_{i}^{(2)}$, repeat the same argument, with $g(s, t)=\left|q_{s} \times q_{t}\right|$ in (3.14). Using the boundedness of $f$ on $U$, we obtain $E_{i}^{(2)}=O\left(1 / n^{2}\right)$. Thus $E_{i}=O\left(1 / n^{2}\right)$, and the sum of the errors over $\left\{\Delta_{1}\right\}$ is $O(1 / n)$.

\section{Evaluation of a singular integral}

The use of integral equations in the solution of potential theory problems in $\mathbf{R}^{3}$ leads to the evaluation of singular surface integrals; for example, see Jaswon and Symm [5]. As an example of the treatment of such integrals, we will consider the evaluation of

$$
\int_{S} d(B) \frac{\partial}{\partial \nu(A)}\left[\frac{1}{|B-A|}\right] d \sigma(A), \quad B \in S,
$$

with $S$ a smooth boundary surface for a simply connected region, $\nu(A)$ the inner normal to $S$ at $A$, and $d(B)$ a smooth density function (called the double layer density). This integral arises from the representation of harmonic functions as double layer potentials in $\mathbf{R}^{3}$; the singularity in (4.1) is of order $1 /|A-B|$. Unfortunately, there does not seem to be any way to remove the singularity using a change of variables, and it must be treated directly. There is another formulation in terms of solid angles: for example, see Mikhlin [10, page 349], but that too has significant problems when trying to calculate the solid angles, especially when $B$ is near to $A$.

As before, assume there is a 1-1 mapping of $U$ onto $S$ and then use it to change the integral in (4.1) to one over $U$. This leads to the integral operator

$$
\mathcal{K} \rho(P)=\int_{U} K(P, Q) \rho(Q) d \sigma(Q), \quad P \in U .
$$

The kernel $K$ includes the original kernel of (4.1) and the change of the surface area differential.

A Product Gaussian Quadrature formula. Begin by applying the wellknown identity

$$
2 \pi=\int_{S} \frac{\partial}{\partial \nu(B)}\left[\frac{1}{|A-B|}\right] d \sigma(B), \quad A \in S
$$

to obtain

$$
\mathcal{K} \rho(P)=2 \pi \rho(P)+\int_{U} K(P, Q)[\rho(Q)-\rho(P)] d \sigma .
$$

The new integrand is bounded at $Q=P$, although it will still be discontinuous. 
If we were now to change to spherical coordinates for $Q$, then the point $P$ would become a singular point internal to the integration region $[0, \pi] \times[0,2 \pi]$. Most numerical integration methods perform poorly in such a situation, and that will be true here. To avoid this, we first rotate the coordinate system,

$$
Q^{\prime}=H Q
$$

with $H$ a Householder orthogonal matrix. It is to be chosen so that $P^{\prime}=H P$ is $(0,0,1)$ or $(0,0,-1)$. We use a spherical coordinates representation for $Q^{\prime}$, and apply the product Gaussian quadrature with respect to this new representation. The singularity in the integrand occurs along either $\theta=0$ or $\theta=\pi$. This change of variables results in much improved accuracy and, moreover, there is now a regular behaviour to the error as the integration parameter $m$ is increased.

EXAmple. (1) We choose $S=U$ and, to have a test case, use the result that $\mathscr{K} \rho=2 \pi /(2 k+1) \rho$, for $k \geqslant 0$, for any spherical harmonic of degree $k$. (See [9, page 69] for the definition of spherical harmonics). We choose the spherical harmonics

$$
\rho^{(1)}(P)=z \quad \text { and } \quad \rho^{(2)}(P)=z^{2}-\left(x^{2}+y^{2}\right) / 2 .
$$

For $P$, we have

$$
(-.30353, .93417, .18759) \text {. }
$$

The index of integration is $m$, and the number of nodes is $n=2 m^{2}$. The numerical results are given in Table 7 .

TABLE 7

Gaussian rule: example 1 for singular integral

\begin{tabular}{|rr|cc|cc|}
\hline$m$ & $n$ & Error for $\mathcal{K} \rho^{(1)}$ & Ratio & Error for $\mathcal{K} \rho^{(2)}$ & Ratio \\
\hline 4 & 32 & $1.4 \mathrm{E}-3$ & 6.9 & $-1.0 \mathrm{E}-2$ & 7.1 \\
8 & 128 & $2.0 \mathrm{E}-4$ & 7.4 & $-1.4 \mathrm{E}-3$ & 7.4 \\
16 & 512 & $2.7 \mathrm{E}-5$ & & $-1.9 \mathrm{E}-4$ & 7.7 \\
32 & 2048 & $3.5 \mathrm{E}-6$ & 7.7 & $-2.5 \mathrm{E}-5$ & \\
\hline
\end{tabular}

(2) We use the surface $S_{3}$ with the density functions of (4.6) and let $P$ be the point on $S_{3}$ corresponding to the point of (4.7) on $U, P=$ $(-.20036,1.23330, .12383)$. The errors in Table 8 were calculated using a very accurate value of $\mathscr{K} \rho(P)$, obtained with a much larger value of $m$. 
TABLE 8

Gaussian rule: example 2 for singular integral

\begin{tabular}{|rr|cc|cc|}
\hline$m$ & $n$ & Error for $\mathcal{K} \rho^{(1)}$ & Ratio & Error in $\mathcal{K} \rho^{(2)}$ & Ratio \\
\hline 4 & 32 & $-5.2 \mathrm{E}-2$ & 92 & $5.6 \mathrm{E}-2$ & -93 \\
8 & 128 & $-5.7 \mathrm{E}-4$ & 11 & $-6.0 \mathrm{E}-4$ & 20 \\
16 & 512 & $-5.4 \mathrm{E}-5$ & & $-3.0 \mathrm{E}-5$ & 7.4 \\
32 & 2048 & $-7.2 \mathrm{E}-6$ & 7.4 & $-4.0 \mathrm{E}-6$ & \\
\hline
\end{tabular}

In these examples and with all other examples we calculated, the error has the asymptotic form

$$
c /\left(m^{3}\right)+O\left(1 / m^{4}\right)
$$

As the surface becomes more ill-behaved, the value of $m$ must be larger before the behaviour (4.8) becomes apparent. With surfaces $S_{2}$ and $S_{4}$, this happens with $m=64$, but the error is still quite small with smaller values of $m$. With an error of the form (4.8), Richardson extrapolation can be used to accelerate the convergence, and that should be an important tool with these singular problems. A similar strategy is suggested by Lyness [8].

A finite ELEMENT integration Formula. Most numerical methods for evaluating (4.1) are based on first writing it as a sum of smaller integrals, based on some triangulation of $S$,

$$
\sum_{j=1}^{n} \int_{\Delta,} d(B) \frac{\partial}{\partial \nu(B)}\left[\frac{1}{|A-B|}\right] d \sigma(B)
$$

Usually each integral over $\Delta_{j}$ is approximated by the central rule, and the surface area of $\Delta$, is approximated by the area of the planar triangle determined by the vertices of $\Delta_{j}$. For examples, see Jaswon and Symm [5, page 233], Birtles et al. [3], and Wait [14, page 303]; the last paper also uses a quadratic isoparametric method, with improved results. This is a very flexible approach to the evaluation of integrals like (4.1), but it also leads to greater inaccuracy than if the special nature of the kernel and surface were taken into account.

We approximate (4.2) using the centroid rule, with a crude modification to avoid elements containing the singular point $P$, using 


$$
\begin{aligned}
\mathcal{K} \rho(P) \approx & \sum_{P \notin \Delta,} K\left(P, Q_{j}\right) \rho\left(Q_{j}\right) \operatorname{Area}\left(\Delta_{j}\right) \\
& +\left[2 \pi-\sum_{P \notin \Delta,} K\left(P, Q_{j}\right) \operatorname{Area}\left(\Delta_{j}\right)\right] \rho(V),
\end{aligned}
$$

where $Q_{j}$ is the centroid of $\Delta_{j}$, and $V$ is some centrally located point in the union of all elements $\Delta_{j}$ containing $P$. The approximation uses (4.3). Empirically, the behaviour of this method is best when $P$ is a centroid of one of the triangles $\Delta_{j}$.

Example. (1) We let $S=U$, and let $\rho$ and $P$ be given by (4.6) and (4.7). The triangulation $T_{i, n}$ is used, and $P$ is a centroid of one of the faces of $T_{i, 20}$. The results are given in Table 9.

TABLE 9

Centroid rule: example 1 for singular integral

\begin{tabular}{|r|cc|cc|}
\hline$n$ & Error in $\mathcal{K} \rho^{(1)}$ & Ratio & Error in $\mathcal{K} \rho^{(2)}$ & Ratio \\
\hline 20 & $-5.7 \mathrm{E}-3$ & 6.1 & $4.2 \mathrm{E}-2$ & 6.2 \\
80 & $-9.4 \mathrm{E}-4$ & 7.0 & $6.8 \mathrm{E}-\mathrm{e}$ & 7.0 \\
320 & $-1.4 \mathrm{E}-4$ & 7.1 & $9.8 \mathrm{E}-4$ & 7.1 \\
1280 & $-1.9 \mathrm{E}-5$ & 6.7 & $1.4 \mathrm{E}-4$ & 6.6 \\
5120 & $-2.9 \mathrm{E}-6$ & & $2.1 \mathrm{E}-5$ & \\
\hline
\end{tabular}

(2) We use the same surface $S_{3}$, density $\rho$, and point $P$ as with example 2 for product Gaussian quadrature. The results are given in Table 10.

TABLE 10

Centroid rule: example 2 for singular integral

\begin{tabular}{|r|cc|cc|}
\hline$n$ & Error in $\mathcal{K} \rho^{(1)}$ & Ratio & Error in $\mathcal{K} \rho^{(2)}$ & Ratio \\
\hline 20 & $1.6 \mathrm{E}-1$ & -2.7 & 1.2 & -83 \\
80 & $-5.9 \mathrm{E}-2$ & -3.7 & $-1.4 \mathrm{E}-2$ & -4.0 \\
320 & $1.6 \mathrm{E}-2$ & -3.8 & $3.5 \mathrm{E}-3$ & -28 \\
1280 & $-4.1 \mathrm{E}-3$ & -4.0 & $-1.3 \mathrm{E}-4$ & -.7 \\
5120 & $1.0 \mathrm{E}-3$ & & $1.9 \mathrm{E}-4$ & \\
\hline
\end{tabular}


As can be seen, the error is quite regular when $S=U$, but not otherwise. This has been borne out with all other examples computed to date. Empirically, for $S=U$, the error is about $O\left(1 / n^{1.5}\right)$, which is equivalent to the result (4.8) for product Gaussian quadrature. There seems to be a fortuitous cancellation of errors taking place in this case. For all other surfaces, the uniform rate is mostly lost.

To compare the two methods of evaluating (4.2), the product Gaussian formula has proved much more regular in convergence than the centroid rule and, usually, the Gaussian formula has been much more accurate as well. In our programs, the Gaussian formula has required less memory storage, the programs have been simpler, and generally it has performed much more efficiently.

\section{Acknowledgement}

This research was supported in part by the National Science Foundation grant MCS-8002422.

\section{References}

[1] K. Atkinson, "The numerical solution of Laplace's equation in three dimensions", SIAM J. Numer. Anal. (in press).

[2] K. Atkinson, "The numerical solution of Laplace's equation in three dimensions, part II", in J. Albrecht and L. Collatz (eds.), Numerical treatment of integral equations (BirkhauserVerlag, Basel, 1980), 1-23.

[3] A. Birtles, B. Mayo, and A. Bennett, "Computer technique for solving 3-dimensional electron-optics and capacitance problems", Proc. IEEE 120 (1972), 213-220.

[4] N. Gunter, Potential theory (Ungar, New York, 1967).

[5] M. Jaswon and G. Symm, Integral equation methods in potential theory and elastastatics (Academic Press, London, 1977).

[6] P. Keast and J. diaz, "Quadrature rules for the surface of the s-dimensional sphere", preprint, Univ. of Toronto, 1979.

[7] V. Lebedev, "Quadratures on a sphere", USSR Comp. Math. and Phys. 16 (1970), 10-24.

[8] J. Lyness, "An error functional expansion for $N$-dimensional quadrature with an integrand function singular at a point", Math. Comp. 30 (1976), 1-23.

[9] T. MacRobert, Spherical harmonics (Pergamon Press, London, 1967).

[10] S. G. Mikhlin, Mathematical physics: an advanced course (North-Holland, Amsterdam, 1970).

[11] D. Ragozin, "Constructive polynomial approximation on spheres and projective spaces", Trans. Amer. Math. Soc. 162 (1971), 157-170.

[12] S. L. Sobolev, "Cubature formulas on the sphere invariant under finite groups of rotations", Soviet Math. 3 (1962), 1307-1310.

[13] A. Stroud, Approximate calculation of multiple integrals (Prentice-Hall, New Jersey, 1971). 
[14] R. Wait, "Use of finite elements in multi-dimensional problems in practice", in J. Walsh and M. Delves (eds.), Numerical solution of integral equations (Oxford University Press, 1974), 300-311.

Mathematics Department

University of Iowa

Iowa City

Iowa 52242

U.S.A. 